\title{
Desulfovibrio vulgaris Hildenborough transcriptomic analysis by Restriction fragment functional display (RFFD)
}

\section{Santana and J. Gonzẩlez}

IRAS-CSIC, 11012 Sevilla, spain

Sulphate-reducing bacteria reduce sulphate with electrons from carbon substrate thereby producing hydrogen sulphide. This reduction is anaerobic; however the metabolte activity of SRB in oxac zones is frequently higher than in neighbour anoxic zones. The large tolemotic conservation of energy and ATP some are able to respire oxygen in a process cotpled Hidenborough sequencing of th genome has production. In the case of Destifowbrio mugroductase genes. In order to understand why aerobia confirmed the presence of putative oxygen. oxyde comparative study of gene expression under aerobic and respiration occuss in D.vighars, we propo angen oxidoreductase mutants, focusing on the identification anaerobic conditions, in wild-type and oxy. Genes encoding regulators of oxyen response are of of genes related to the aerobic metabolism, Genes encoding
special interest RFWD technique, here described, constitutes a powerful tool to do such study.

Keywords: Desulfovibrio vilgaris, gene expression, RFID, oxygen tesponse 